\title{
Microfluidic resonators with two parallel channels for independent sample loading and effective density tuning
}

\author{
Jungchul Lee ${ }^{1,2^{*}}$ (D) Faheem Khan ${ }^{3}$, Thomas Thundat ${ }^{4}$ and Bong Jae Lee ${ }^{1,2}$ (])
}

\begin{abstract}
This paper reports doubly clamped microchannel embedded resonators with two independent and parallel channels integrated for effective sample density tuning for the first time. With the aid of such a unique design, each fluidic channel can be independently accessed thus different liquid samples can be loaded simultaneously. The proposed fluidic resonators are batch fabricated by depositing silicon nitride, polysilicon, and silicon nitride sequentially on top of a set of 4-inch silicon wafers and sacrificing the middle polysilicon layer with potassium hydroxide $(\mathrm{KOH})$. The sacrificial process defines two parallel channels and releases doubly clamped beam resonators simultaneously. In addition, an off-chip vacuum clamp with optical and fluidic access is custom-made to operate each resonator with enhanced quality factor. The microfluidic resonators mounted on the custom vacuum clamp are thoroughly characterized with a laser Doppler vibrometer and used to measure the effective sample density ranging from 395 to $998 \mathrm{~kg} / \mathrm{m}^{3}$.
\end{abstract}

Keywords: Batch fabrication, Density sensing, Density tuning, Microfluidic resonator, Sacrificial process, Vacuum clamp

\section{Background}

Microfluidic resonators including suspended microchannel resonators (SMRs) [1] and hollow microtube resonators (HMRs) [2, 3] have been applied for measurements of liquids, synthetic particles, and biological matters including cells, bacteria, virus, and exosomes with the outstanding performance. However, the fabrication process of such promising microfluidic resonators is yet laborious thus expensive since the majority of related research effort is focused on further improvement of device performance, mostly resolution, by making smaller resonators or achieving high quality factor via wafer-level on-chip vacuum packaging. In addition, only a single channel or bifurcated channels are embedded within resonators to date which limit the independent

\footnotetext{
*Correspondence: jungchullee@kaist.ac.kr

1 Department of Mechanical Engineering, Korea Advanced Institute of Science and Technology, 291 Daehak-ro, Yuseong-gu, Daejeon 34141, Republic of Korea

Full list of author information is available at the end of the article
}

injection and characterization of multiple samples simultaneously. This may particularly be useful in an industrial application where a calibration standard needs to be present in the sensor all the time while samples of interest are tested. Additionally, such an arrangement also helps in achieving higher accuracy through the concept of differential measurements of density. Therefore, multiple samples of interest must be tested sequentially one at a time and this, in turn, increases the overall analysis time, risk of contamination, and inconvenience in calibration thus results in higher operation and maintenance costs. Moreover, mass densities of liquids are a few orders of magnitude larger than those of gases, the intermediate range of sample density is not available with microfluidic resonators with a single embedded channel [4-6]. In this paper, we propose simple fabrication of microfluidic resonators with two parallel channels and demonstrate independent sample loading and effective sample density tuning. 


\section{Experimental details}

\section{Fabrication}

Figure 1 shows design and fabrication processes of the proposed doubly clamped microfluidic resonators with two embedded parallel channels. Proposed microfluidic resonators are batch fabricated with a set of 4-inch silicon wafers by deposition, etching, and sacrificial process $[7,8]$. Silicon nitride and polycrystalline silicon are sequentially deposited by low pressure chemical vapor deposition (LPCVD). Next, regions for two parallel channels of microfluidic resonators are patterned by photolithography and reactive ion etching on the polysilicon layer. Then, another LPCVD silicon nitride layer is deposited on the patterned polysilicon and previously deposited silicon nitride layer. The secondly deposited silicon nitride layer defines channel sidewalls and top covers of microfluidic resonators. Finally, patterned polysilicon regions are sacrificially removed by potassium hydroxide $(\mathrm{KOH})$ etching and microfluidic resonators are released at the same time. Two parallel microchannels (length of $500 \mu \mathrm{m}$, width of $16 \mu \mathrm{m}$, and height of $3 \mu \mathrm{m}$ ) are integrated on top of a plain doubly clamped beam (length of $500 \mu \mathrm{m}$, width of $56 \mu \mathrm{m}$, and height of $0.4 \mu \mathrm{m})$. Fundamental resonance frequency of fabricated resonators with two parallel microchannels is calculated to be $218.8 \mathrm{kHz}$. Larger sample delivery and waste collection channels are connected with two channels embedded in the microfluidic resonator and four liquid ports that are configured during the polysilicon sacrificial etching. Figure 2 shows optical and scanning electron micrographs of a fabricated doubly clamped microfluidic resonator with two embedded channels. The scanning electron micrograph in Fig. $2 \mathrm{~d}$ shows an intentionally damaged resonator near its clamping region that confirms well-defined parallel channel structures.

\section{Device mount}

In order to measure the mechanical resonance frequency (with high quality factor) of the microfluidic resonators, a custom off-chip vacuum clamp is designed and made using a precision CNC milling machine. The plug-n-play style vacuum clamp made it highly practical to exchange the sensors after each measurement. Figure 3a shows the 3D CAD of the custom off-chip vacuum clamp composed of two (top and bottom) parts. The top part exhibits a transparent glass window for optical access and a gas vent hole connected to a standard KF16 vacuum flange. The bottom part exhibits four microfluidic ports, machined regions for four O-rings, the resonator chip, and the piezo actuator, respectively. Stainless steel tubes connected to the four microfluidic

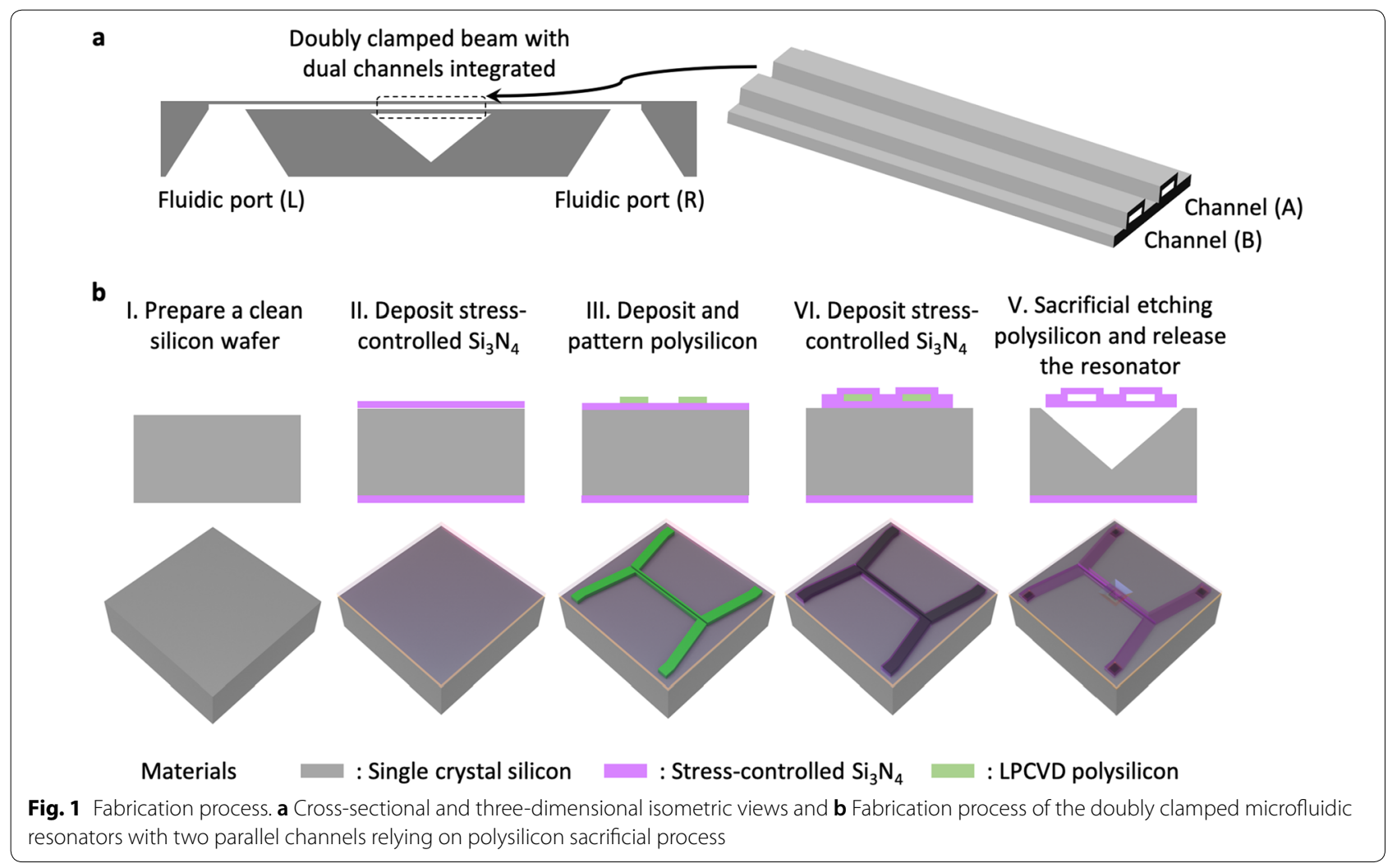



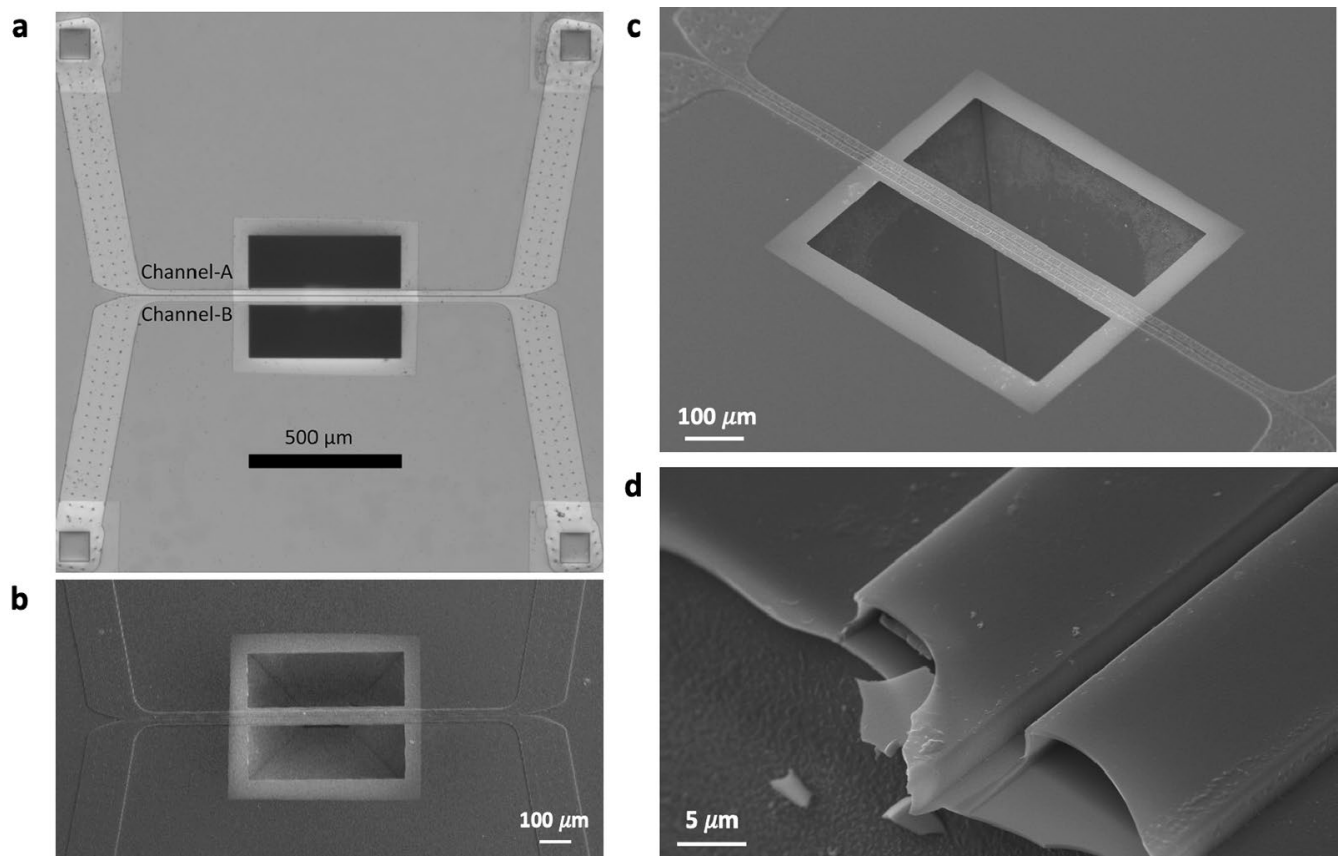

Fig. 2 Optical and scanning electron micrographs of the fabricated microfluidic resonators with two parallel channels. a Top view optical micrograph. $\mathbf{b}$ Top and $\mathbf{c}$ isometric view scanning electron micrographs. $\mathbf{d}$ Scanning electron micrograph showing the interior of two parallel channels of an intentionally broken device

ports simply become off-chip bypass channels that facilitate the liquid sample loading and exchange. Figure $3 \mathrm{~b}$ shows the bottom part with four stainless steel tubes inserted (top), with four O-rings placed (middle), and with a microfluidic resonator placed (bottom), respectively. Once four O-rings and a resonator chip are placed in the bottom part, it is tightened with the top part by using four screws and four wing nuts while a larger O-ring is inserted between the two parts as shown in Fig. 3c. The resonator chip firmly fixed between one larger O-ring on top and four small O-rings at bottom prevents leakage during the operation of a turbo pump attached to the KF16 vacuum flange.

\section{Measurements}

To test the custom-made vacuum clamp, amplitude and phase responses for a microfluidic resonator (with air present in the both channels) are collected with a laser doppler vibrometer (MSA-500, Polytec) at partial vacuum as well as atmospheric pressure. Figures $4 \mathrm{a}$ and $\mathrm{b}$ show data for 0.062 mbar where the fundamental resonance frequency and corresponding quality factor are $218.8 \mathrm{kHz}$ and 10,940 , respectively. Figure $4 \mathrm{c}$ shows the quality factor as a function of the vacuum level and
Fig. 4d shows the maximum amplitude at the fundamental resonance frequency as a function of the vacuum level. At $1 \mathrm{~atm}$ and $6.1 \times 10^{-5} \mathrm{mbar}$, resonance frequencies are 209.2 and $218.9 \mathrm{kHz}$, respectively and quality factors are 186 and 15,094, respectively. Both the quality factor and the maximum amplitude are significantly increased under vacuum condition.

Next, the microfluidic resonator is characterized while its two channels are filled with different liquid samples. Figure 5a shows the resonance frequency shift when two parallel channels are partially or fully filled with ethanol or water with referenced to the case when both channels are empty (filled with air). For a same pair of samples, four scenarios are possible upon sample loading considering duplication. When injection channels for two different samples are switched, there are slight mismatches in the resonance frequency shift observed at around $-24,-29$, and $-46 \mathrm{kHz}$. Those discrepancies are attributed to the fabrication tolerance. Figure $5 \mathrm{~b}$ represents the resonance frequency shift as a linear function of the average density, $\left(\rho_{A}+\rho_{B}\right) / 2$. With ethanol and air, the effective sample density as low as $395 \mathrm{~kg} / \mathrm{m}^{3}$ is easily configured. As expected, further down tuning of the effective sample density is enabled by adding more embedded channels. 


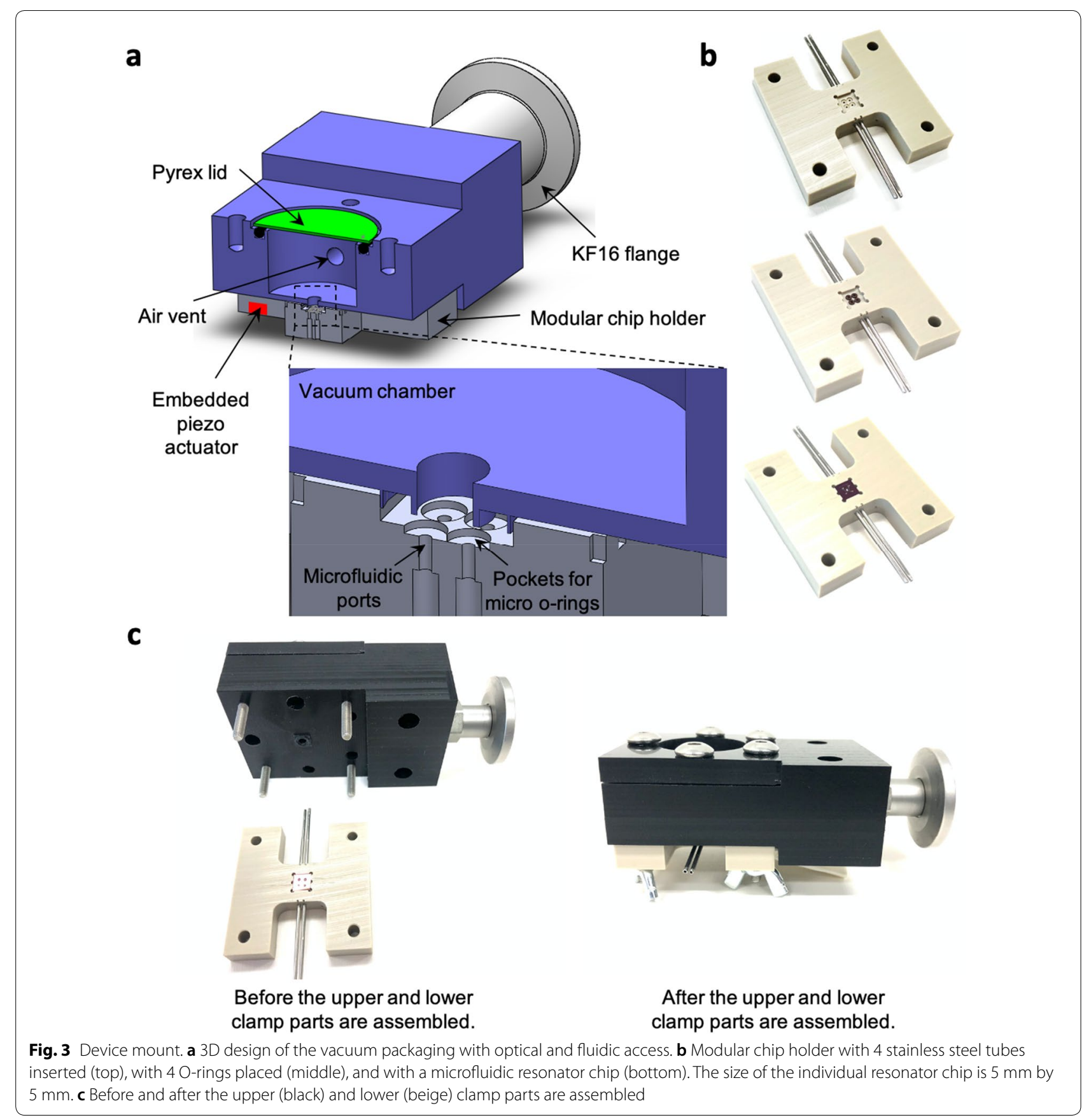

\section{Conclusions}

This paper reports microfabrication of doubly clamped beam resonators with two parallel embedded channels that can be injected with identical or different liquid samples or selectively emptied. Especially, the effective sample density can be significantly lowered by injecting a liquid sample into only one channel. The custom offchip vacuum clamp introduced herein simply realizes 

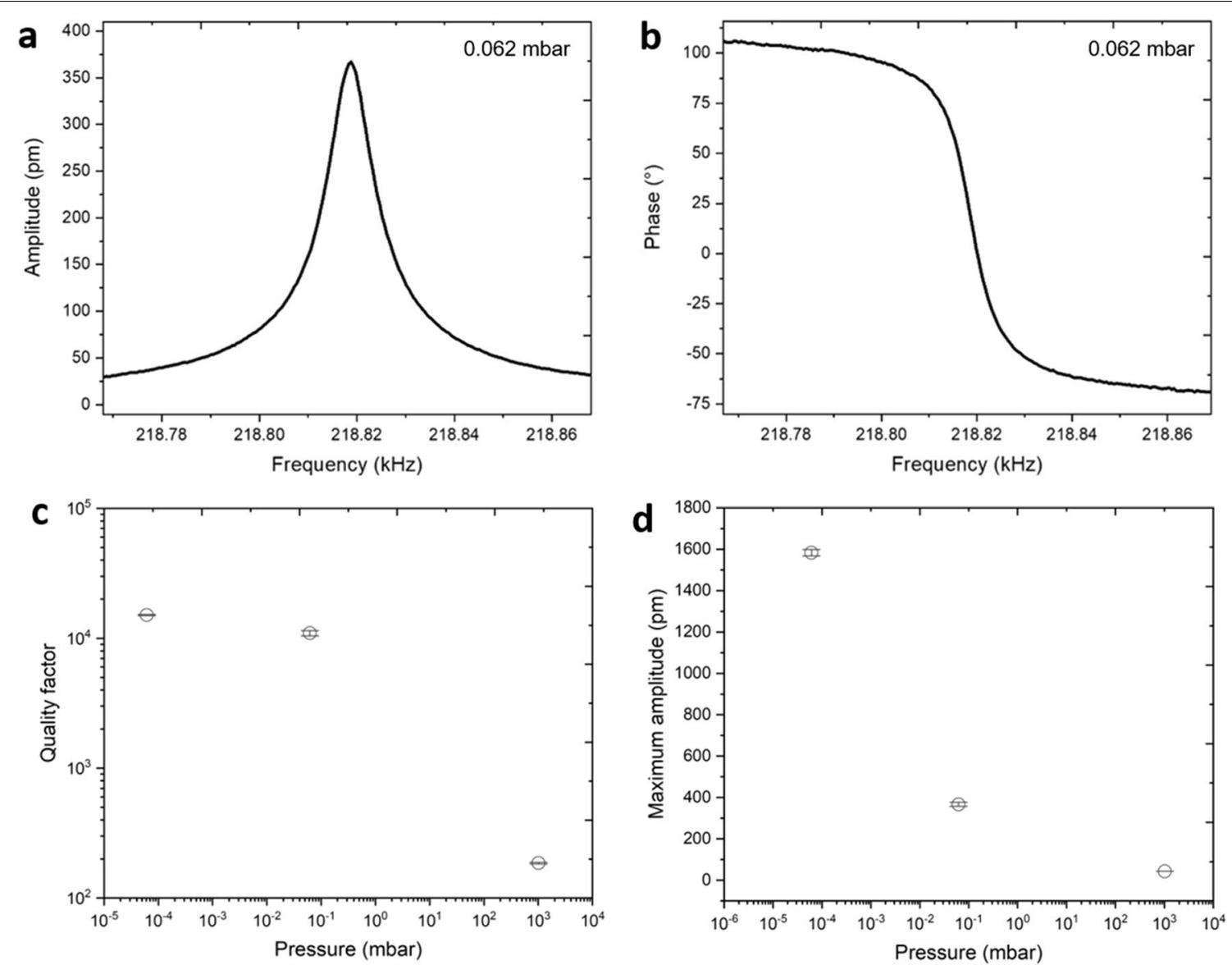

Fig. 4 Resonance spectra and pressure dependence. a Amplitude and $\mathbf{b}$ phase spectra of a microfluidic resonator with two parallel channels filled with air measured at 0.062 mbar. c Quality factor as a function of the vacuum level. Standard deviations are 2.85, 518.59, and 143.17 for 1013, 0.062, and $6.1 \times 10^{-5} \mathrm{mbar}$, respectively. $\mathbf{d}$ Maximum amplitude at the fundamental resonance as a function of the vacuum level. Standard deviations are $0.22,9.92$, and $15.12 \mathrm{pm}$, respectively

two bypass channels that facilitate and expedite sample loading and exchange while providing dissipation-minimized environment. Microchannel resonators reported in this paper can be employed for several interesting applications. For example, if two channels are filled with two liquids at different temperatures, microscale heat exchange experiments can be performed in both parallel and counter flow configurations. If one of two channels is filled with plasmonic or magnetic particle suspensions, remote photothermal or induction heating can be enabled so that an actual liquid sample introduced into the remaining channel can be interrogated at various modulated temperatures. 

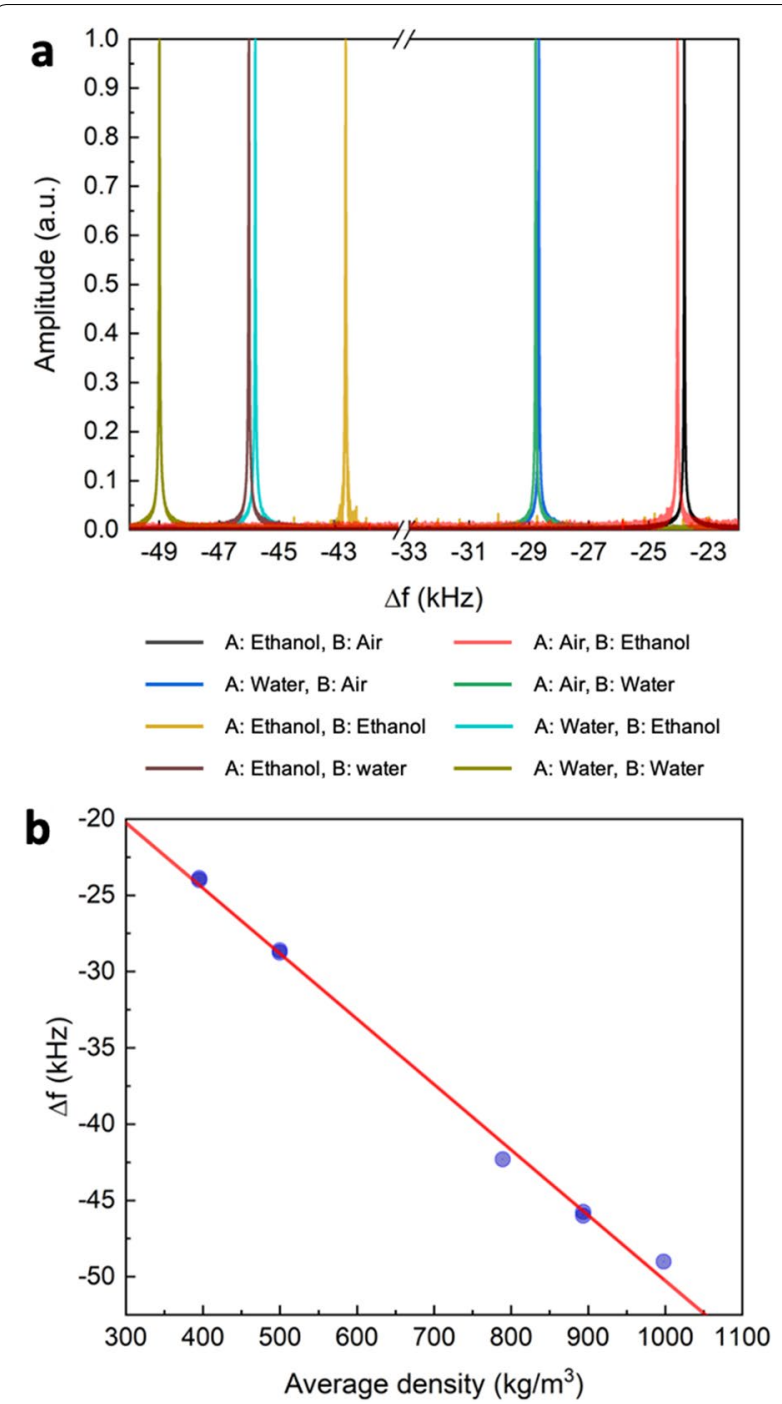

Fig. 5 Density measurements. a Resonance frequency shift when two parallel channels are partially or fully filled with ethanol or DI water with referenced to the case when both channels are empty. b Resonance frequency shift as a function of the average sample density

\section{Acknowledgements}

Not applicable.

\section{Authors' contributions}

$J \mathrm{~L}$ and FK developed the idea and performed the experiments. $J \mathrm{~L}$ and FK analyzed the results and all authors drafted the manuscript together. All authors read and approved the final manuscript.

\section{Funding}

This research was supported by the National Research Foundation of Korea (NRF) funded by the Korea government (Ministry of Science and ICT) (NRF2020R1A2C3004885 and NRF-2020R1A4A2002728).

\section{Availability of data and materials}

The datasets used and/or analysed during the current study are available from the corresponding author on reasonable request.

Ethics approval and consent to participate

Not applicable.

\section{Consent for publication}

Not applicable.

\section{Competing interests}

The authors declare that they have no competing interests.

\section{Author details}

${ }^{1}$ Department of Mechanical Engineering, Korea Advanced Institute of Science and Technology, 291 Daehak-ro, Yuseong-gu, Daejeon 34141, Republic of Korea. ${ }^{2}$ Center for Extreme Thermal Physics and Manufacturing, Korea Advanced Institute of Science and Technology, Daejeon 34141, Republic of Korea. ${ }^{3}$ Fourien, Edmonton T6B2N2, Canada. ${ }^{4}$ Department of Chemical and Biological Engineering, University at Buffalo, New York 14260-4200, USA.

Received: 31 July 2020 Accepted: 24 September 2020

Published online: 03 October 2020

\section{References}

1. Burg T, Godin M, Knudsen SM, Shen W, Carlson G, Foster JS, Babcock K, Manalis SR (2007) Weighing of biomolecules, single cells and single nanoparticles in fluid. Nature 446:1066-1069

2. Kim J, Song J, Kim K, Kim S, Song J, Kim N, Khan MF, Zhang L, Sader JE, Park K, Kim D, Thundat T, Lee J (2016) Hollow microtube resonators via silicon self-assembly toward subattogram mass sensing applications. Nano Lett 16(3):1537-1545

3. Je J, Song J, Lee B, Lee J (2019) Multiscale fluidic channels via internal oxidation and oxide etching of self-assembled silicon-on-nothing structures. J Microelectromech Syst 28(5):865-868 
4. Sparks D, Smith R, Straayer M, Cripe J, Schneider R, Chimbayo A, Anasari S, Najifi N (2003) Measurement of density and chemical concentration using a microfluidic chip. Lab Chip 3:19-21

5. Khan MF, Schmid S, Larsen PE, Davis ZJ, Yan W, Stenby EH, Boisen A (2013) Online measurement of mass density and viscosity of $\mathrm{pL}$ fluid samples with suspended microchannel resonator. Sens. Actuators, B-Chem. 185:456-461

6. Lee I, Park K, Lee J (2013) Precision density and volume contraction measurements of ethanol-water binary mixtures using suspended microchannel resonators. Sens. Actuators, A-Phys. 194:622-666

7. Khan MF, Schmid S, Davis ZJ, Dohn S, Boisen A (2011) Fabrication of resonant cantilevers with integrated transparent fluidic channel. Microelectron Eng 88:2300-2303
8. Scaiola D, Stassi S, Calmo R, Maillard D, Varricchio SSG, Pastina AD, Villanueva G, Renaud P, Ricciardi C (2020) Fabrication of clamped-clamped beam resonators with embedded fluidic nanochannel. Microelectron Eng 231:111395

\section{Publisher's Note}

Springer Nature remains neutral with regard to jurisdictional claims in published maps and institutional affiliations.

\section{Submit your manuscript to a SpringerOpen ${ }^{\circ}$ journal and benefit from:}

- Convenient online submission

- Rigorous peer review

- Open access: articles freely available online

- High visibility within the field

- Retaining the copyright to your article

Submit your next manuscript at $\boldsymbol{\nabla}$ springeropen.com 